\title{
The Hans algorithm is not prognostic in patients with diffuse large B-cell lymphoma treated with R-CHOP
}

\author{
Jorge J. Castillo ${ }^{\mathrm{a}, *}$, Brady E. Beltran ${ }^{\mathrm{b}}$, Moo-Kon Song ${ }^{\mathrm{c}}$, Ivana Ilic $^{\mathrm{d}}$, Sirpa Leppa ${ }^{\mathrm{e}}$, Heidi Nurmi ${ }^{\mathrm{e}}$, \\ Ritsuko Seki ${ }^{\mathrm{f}}$, Silvia Uccella ${ }^{\mathrm{g}}$, Jun-Min $\mathrm{Li}^{\mathrm{h}}{ }^{\text {, Diana O. Treaba }}{ }^{\mathrm{i}}$, Dariusz Stachurski ${ }^{\mathrm{i}}$, James N. Butera ${ }^{\mathrm{j}}$ \\ a Division of Hematology and Oncology, The Warren Alpert Medical School of Brown University, The Miriam Hospital, Providence, RI, USA \\ b Department of Oncology and Radiotherapy, Hospital Nacional Edgardo Rebagliati Martins, Lima, Peru

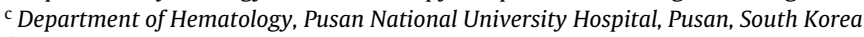 \\ d Department of Pathology and Cytology, University Hospital Center Zagreb, Zagreb, Croatia \\ e Department of Oncology, Helsinki University Central Hospital, Helsinki, Finland \\ ${ }^{\mathrm{f}}$ Kyushu Lymphoma Study Group, Kurume University School of Medicine, Kurume, Japan \\ ${ }^{g}$ Department of Surgical and Morphological Sciences, Pathology Unit, University of Insubria, Varese, Italy \\ ${ }^{\mathrm{h}}$ Department of Hematology, Shanghai Jiaotong University School of Medicine, Ruijin Hospital, Shanghai, China \\ i Department of Pathology, The Warren Alpert Medical School of Brown University, Rhode Island Hospital, Providence, RI, USA \\ ${ }^{j}$ Division of Hematology and Oncology, The Warren Alpert Medical School of Brown University, Rhode Island Hospital, Providence, RI, USA
}

\section{A R T I C L E I N F O}

\section{Article history:}

Received 31 May 2011

Received in revised form

13 December 2011

Accepted 14 December 2011

Available online 24 January 2012

\section{Keywords:}

Diffuse large B-cell lymphoma

Non-germinal center

Rituximab

Hans algorithm

\begin{abstract}
A B S T R A C T
Our objective was to evaluate the non-germinal center (GC) profile as a marker for response and survival in DLBCL and to compare the characteristics of patients with GC and non-GC DLBCL treated with rituximab-containing regimens. In this patient-level meta-analysis, retrospective data from 712 newly diagnosed DLBCL patients treated with chemoimmunotherapy from 7 centers were analyzed. GC and non-GC profiles were defined according to the Hans algorithm. Although the non-GC profile showed a trend towards worse overall survival (HR $1.24,95 \% \mathrm{CI} 0.92-1.66 ; p=0.15$ ) and progression-free survival (HR 1.29, 95\% Cl 0.96-1.73; $p=0.09$ ), it did not retain its value in the multivariate survival analysis. Additionally, the non-GC profile was independently associated with worse complete response rates (OR 0.55, $95 \%$ CI $0.37-0.83 ; p<0.01)$ in the multivariate logistic regression analysis. Interestingly, Asian patients had higher proportion of GC DLBCL $(p=0.01)$.
\end{abstract}

(C) 2011 Elsevier Ltd. All rights reserved.

\section{Introduction}

Diffuse large B-cell lymphoma (DLBCL) is the most common subtype of non-Hodgkin lymphoma (NHL), accounting for approximately $30-40 \%$ of the cases seen worldwide [1]. Despite its aggressive clinical course, DLBCL is considered a curable disease and, within the last years, the addition of rituximab to chemotherapy has further increased the rates of complete response (CR) as well as the median overall survival (OS) of patients with newly diagnosed DLBCL $[2,3]$. The combination of rituximab, cyclophosphamide, doxorubicin, vincristine and prednisone (R-CHOP) is considered the standard of care for patients with DLBCL. However, given the molecular heterogeneity of DLBCL [4], it is not surprising

\footnotetext{
* Corresponding author at: Division of Hematology and Oncology, The Miriam Hospital, 164 Summit Ave, Providence, RI 02906, USA. Tel.: +1 401793 7151; fax: +1 4017937132 .

E-mail address: jcastillo@lifespan.org (J.J. Castillo).
}

there is also a marked clinical variability. In fact, the prognosis of patients with DLBCL varies greatly depending on their risk strata.

Since 1993, the International Prognostic Index (IPI) score has largely been considered the standard for risk-stratification of patients with aggressive variants of DLBCL [5]. More recently, with further refinement of molecular diagnostic methods, at least two variants of DLBCL have been identified as germinal center B-celllike and activated B-cell-like DLBCL; the latter been associated with a worse outcome in patients treated with chemotherapy [6,7]. There have been significant obstacles to translate this finding into clinical practice since molecular diagnostic testing such as gene array analysis require specialized laboratory personnel and fresh tissue. In 2004, Hans and colleagues proposed an immunohistochemical (IHC) method to classify patients with DLBCL into germinal center (GC) and non-GC subtypes [8]. In that study, the non-GC subtype was associated with a worse prognosis as compared to the GC subtype in patients treated with chemotherapy; however, the role of the non-GC subtype in the rituximab era has not been completely elucidated. 
The main purpose of this study was to evaluate the predictive and/or prognostic role that the non-GC profile, as described by Hans, could have in newly diagnosed patients with DLBCL treated with R-CHOP. A secondary objective was to compare clinical and pathological characteristics between patients with GC vs. non-GC DLBCL.

\section{Patients and methods}

A literature search from January 2004 to June 2010 was performed using the search key: rituximab and (immunophenotyping or "germinal center" or "activated Bcell"), looking for retrospective studies evaluating the role of the non-GC profile, as defined by Hans, in newly diagnosed adult patients with DLBCL who were treated with R-CHOP given every 3 weeks. Once studies were identified, the authors of each study were contacted to obtain pertinent patient-level data. The data requested included age at diagnosis, sex, performance status according to the Eastern Cooperative Oncology Group (ECOG) scale, lactate dehydrogenase (LDH) levels, number of extranodal sites, Ann Arbor clinical stage, IPI score, expression of CD10, BCL6 and MUM1/IRF4 by the tumor cells, response to chemotherapy, OS in months and final outcome. In addition to the cases submitted by outside researchers, previously untreated adult patients diagnosed with DLBCL from January 2002 to December 2008 and received therapy with R-CHOP were identified from the medical records of The Miriam and Rhode Island Hospitals in Providence, RI. Clinical data as mentioned above were obtained through a retrospective chart review. Patients with primary CNS lymphoma and primary cutaneous, transformed or HIV-associated DLBCL were not included in this study. Pathological samples of the selected patients were retrieved and immunohistochemical staining was performed after deparaffinization in each case. Antibodies, dilution and manufacturers of the immunostains used by each institution are shown in Table 1 . According to the classification proposed by Hans [8], each case was assigned as GC or non-GC; CD10, BCL6 and MUM1/IRF4 expression was considered positive if at least $30 \%$ of the tumor cells stained with the antibody. A second look study was performed to assess potential inter-observer variability of evaluating the expression of CD10, BCL6 and MUM1/IRF4. A total of 65 cases (9\%), 20 from Finland, 18 from Korea and 27 from the US, were independently evaluated by another investigator and correlation coefficients ( $r$ coefficient) were calculated; $r$-values $>0.75$ represent a strong correlation level. The institutional review boards at each of the participating institutions approved the present study.

Clinicopathological characteristics were compared using Mann-Whitney and Chi-square tests for continuous and categorical variables, respectively. Multiple logistic regression analysis was used to evaluate the predictive value of the nonGC profile for response to R-CHOP. OS was defined as the time elapsed between date of diagnosis and date of death or last follow-up. Progression-free survival (PFS) was defined as the time elapsed between date of diagnosis and date of progression, death or last follow-up .The Kaplan-Meier method was used to calculate the survival curves which were compared using the log-rank test. For the multivariate survival analysis, the Cox proportional hazard regression test was used including age, performance status, LDH levels, number of extranodal sites, clinical stage and immunohistochemical profile. All graphics and calculations were obtained using the statistical software MedCalc ${ }^{\circledR}$ version 11 (Mariakerke, Belgium).

\section{Results}

Out of 13 contacted research groups, six submitted patientlevel data [9-14] accounting for 663 patients. Three patients were excluded because they were $<18$ years. Additionally, 52 patients were identified at our institution for a total of 712 newly diagnosed R-CHOP-treated patients with DLBCL.

\subsection{Clinicopathological characteristics}

The median age was 64 years (range $18-90$ years), and 386 cases (54\%) were men. Regarding adverse clinical variables, 433 patients $(61 \%)$ were $>60$ years, 160 (22\%) had a performance status ECOG >1, 381 (54\%) had elevated LDH levels, $112(16 \%)$ had $>1$ extranodal site involved and $372(52 \%)$ had advanced stage at presentation. Based on the IPI score, 241 patients (34\%) had high or high-intermediate scores. According to the Hans classification, 379 patients (53\%) had a GC and 333 (47\%) had a non-GC profile. There were no major differences between the characteristics of GC versus non-GC DLBCL patients with the exception of a higher proportion of GC DLBCL in Asian than in Western patients (68\% vs. $59 \% ; p=0.01$ ).

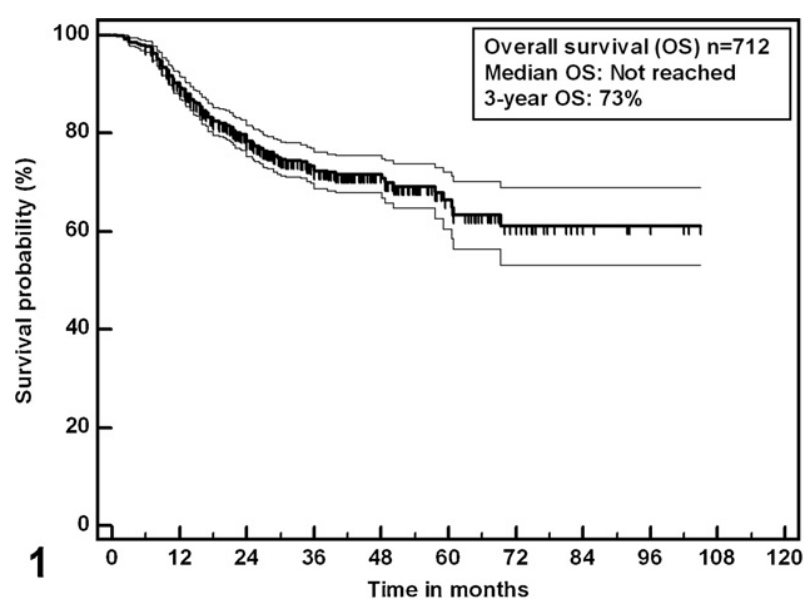

Fig. 1. Overall survival estimates in 712 patients with diffuse large B-cell lymphoma treated with R-CHOP

The comparison between GC and non-GC DLBCL patients treated with R-CHOP is shown in Table 2.

\subsection{Response analyses}

Response data were available in 708 patients; 576 patients (81\%) achieved a complete response (CR), 83 (12\%) a partial response and 49 (7\%) did not respond to R-CHOP. By using multivariate logistic regression, we evaluated age $>60$, male sex, ECOG $>1$, elevated LDH levels, $>1$ extranodal sites, advanced stage and non-GC profile as predictors for CR $(n=700)$. The non-GC profile was associated with an odds ratio (OR) of 0.55 (95\% CI 0.37-0.83; $p<0.01)$. ECOG $>1$ and advanced stage were also independent negative predictive factors for CR with ORs of 0.5 (95\% CI $0.33-0.78 ; p<0.01)$ and 0.44 (95\% CI $0.29-0.68$; $p<0.01)$, respectively. We evaluated the association with overall response rate (ORR) in a similar manner $(n=700)$; ECOG $>1$, elevated LDH levels and advanced stage were independently associated with ORR with ORs of 0.52 (95\% CI $0.27-0.97 ; p=0.03$ ), 0.45 (95\% CI $0.24-0.85 ; p=0.01$ ) and 0.41 (95\% CI $0.21-0.82$; $p=0.01)$, respectively. The non-GC profile was not retained in the model.

\subsection{Survival analysis}

For the entire group ( $n=712$ ), the 3-year and 5-year OS were $73 \%$ and $66 \%$, respectively, and the median os has not yet been reached (Fig. 1). At the univariate level, the non-GC profile showed statistical trends towards being prognostic for OS and PFS with hazard ratios (HRs) of 1.24 (95\% CI 0.92-1.66; $p=0.15$, Fig. 2) and 1.29 (95\% CI 0.96-1.73; $p=0.09$, Fig. 3). At the multivariate level, the non-GC profile was not retained as an independent factor for OS or PFS when compared against the components of the IPI score (data not shown). In subgroup analyses, the non-GC profile was significantly associated with a worse OS in patients with advanced stage with OR of 1.47 (95\% CI 1.05-2.07; $p=0.03$; Fig. 4) and showed a statistical trend towards a worse OS in men and in patients<60years with OR of $1.33(95 \% \mathrm{CI}$ $0.92-1.94 ; p=0.1)$ and OR $1.48(95 \%$ CI $0.92-2.38 ; p=0.1)$, respectively.

\subsection{Pathological correlation}

Our second-look study showed a strong correlation between pathologists when evaluating CD10 with an $r$-value 0.83 (95\% CI 
Table 1

List of antibodies, dilutions and manufacturers of the immunostains used by each institution.

\begin{tabular}{|c|c|c|c|c|}
\hline Institution & Independent validation? & CD10 & BCL6 & MUM1/IRF4 \\
\hline Present & Yes & 56C6; 1:10, Novocastra & PG-B6P; 1:10; Dako & MUM1p; 1:200; Dako \\
\hline Song [9] & Yes & 56C6; 1:20; Lab Vision & PG-B6P; 1:40; Dako & MUM1p; 1:50; Dako \\
\hline Seki [10] & Yes & 56C6; 1:20; Novocastra & P1F6; 1:20; Novocastra & MUM1p; 1:100; Dako \\
\hline Ilic [11] & Yes & $56 C 6 ; 1: 20 ;$ Novocastra & P1F6; 1:20; Novocastra & MUM1p; 1:100; Dako \\
\hline Uccella [12] & Yes & $56 C 6 ; 1: 20 ;$ Novocastra & GI191E/A8; undiluted; Cell Marque & MUM1p; 1:50; Dako \\
\hline Nyman [13] & Yes & $56 C 6 ; 1: 20 ;$ Novocastra & PG-B6P; 1:40; Dako & MUM1p; 1:100; Dako \\
\hline Xia [14] & Yes & $56 C 6 ; 1: 80 ;$ Novocastra & PG-B6P; 1:10; Dako & MUM1p; 1:40; Dako \\
\hline
\end{tabular}

Table 2

Clinicopathological comparison between GC and non-GC DLBCL patients treated with R-CHOP.

\begin{tabular}{|c|c|c|c|c|}
\hline & All patients (\%) & GC DLBCL (\%) & Non-GC DLBCL (\%) & $p$-Value \\
\hline Total & $712(100 \%)$ & $379(53 \%)$ & $333(37 \%)$ & - \\
\hline \multicolumn{5}{|l|}{ Age $(n=712)$} \\
\hline Median age (range) & $64(18-90)$ & $65(18-89)$ & $63(18-90)$ & 0.4 \\
\hline$<60$ years & $279(39 \%)$ & $153(40 \%)$ & $126(38 \%)$ & 0.54 \\
\hline 60 years or older & $433(61 \%)$ & $226(60 \%)$ & $207(62 \%)$ & \\
\hline \multicolumn{5}{|l|}{$\operatorname{Sex}(n=712)$} \\
\hline Female & $326(46 \%)$ & $179(47 \%)$ & $147(44 \%)$ & 0.45 \\
\hline Male & $386(54 \%)$ & $200(53 \%)$ & $186(56 \%)$ & \\
\hline \multicolumn{5}{|l|}{ Ethnicity $(n=712)$} \\
\hline Asian & $455(64 \%)$ & $259(68 \%)$ & $196(59 \%)$ & 0.01 \\
\hline Western & $257(36 \%)$ & $120(32 \%)$ & $137(41 \%)$ & \\
\hline \multicolumn{5}{|l|}{ Performance $(n=709)$} \\
\hline ECOG $<2$ & $549(77 \%)$ & $298(79 \%)$ & $251(76 \%)$ & 0.47 \\
\hline ECOG 2 or higher & $160(23 \%)$ & $81(21 \%)$ & $79(24 \%)$ & \\
\hline \multicolumn{5}{|l|}{ LDH levels $(n=707)$} \\
\hline Normal & $326(46 \%)$ & $171(46 \%)$ & $155(47 \%)$ & 0.83 \\
\hline Elevated & $381(54 \%)$ & $204(54 \%)$ & $177(53 \%)$ & \\
\hline \multicolumn{5}{|l|}{ Extranodality $(n=709)$} \\
\hline$<2$ sites & $597(84 \%)$ & $328(86 \%)$ & $269(81 \%)$ & 0.08 \\
\hline 2 sites or more & $112(16 \%)$ & $52(14 \%)$ & $61(19 \%)$ & \\
\hline \multicolumn{5}{|l|}{ Stage $(n=712)$} \\
\hline Early stage & $340(48 \%)$ & $192(51 \%)$ & $148(44 \%)$ & 0.11 \\
\hline Advanced stage & $372(52 \%)$ & $187(49 \%)$ & $185(56 \%)$ & \\
\hline \multicolumn{5}{|l|}{ IPI score $(n=712)$} \\
\hline Low & $332(47 \%)$ & $186(49 \%)$ & $146(44 \%)$ & 0.15 \\
\hline Low-intermediate & $139(20 \%)$ & $72(19 \%)$ & $67(20 \%)$ & \\
\hline High-intermediate & $104(15 \%)$ & $54(14 \%)$ & $50(15 \%)$ & \\
\hline High & 137 (19\%) & $67(18 \%)$ & $70(21 \%)$ & \\
\hline
\end{tabular}

DLBCL, diffuse large B-cell lymphoma; ECOG, Eastern Cooperative Oncology Group; GC, germinal center; IPI, International Prognostic Index; LDH, lactate dehydrogenase.

0.73-0.89). The level of correlation for MUM1/IRF4 and BCL6 was less strong with $r$-values 0.67 (95\% CI $0.51-0.79$ ) and 0.58 (95\% CI 0.46-0.86), respectively. However, when evaluating the Hans algorithm, there was a strong inter-observer correlation with an r-value 0.85 (95\% CI 0.76-0.90).

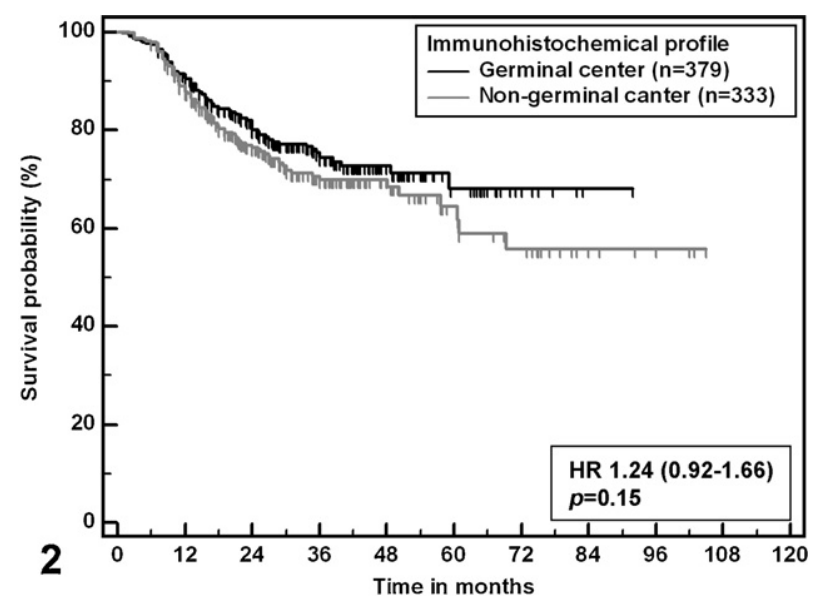

Fig. 2. Overall survival estimates in 712 patients with diffuse large B-cell lymphoma treated with R-CHOP, according to the Hans algorithm.

\section{Discussion}

Molecular differentiation by microarray analysis of patients with DLBCL between GC and non-GC subtypes are of prognostic value in the rituximab era [4]. However, a reliable differentiation of these two subtypes using IHC has historically been challenging

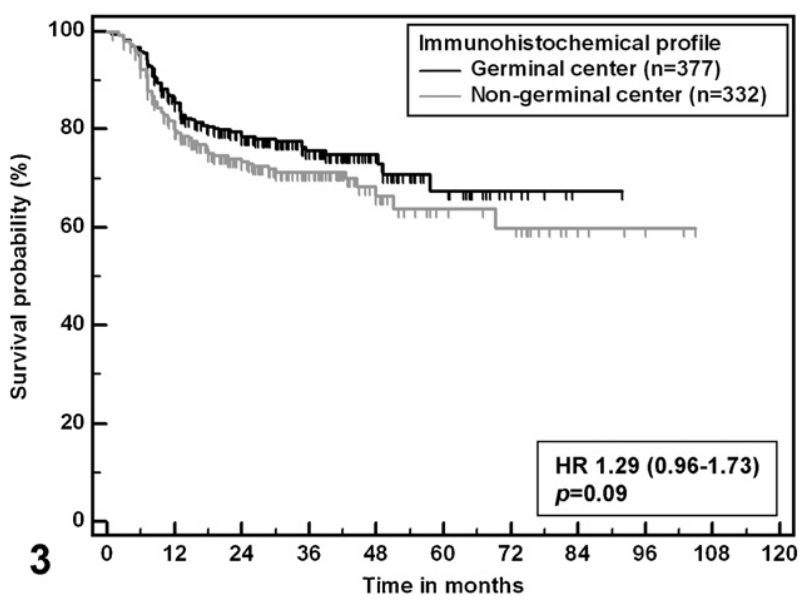

Fig. 3. Progression-free survival estimates in 712 patients with diffuse large B-cell lymphoma treated with R-CHOP, according to the Hans algorithm. 


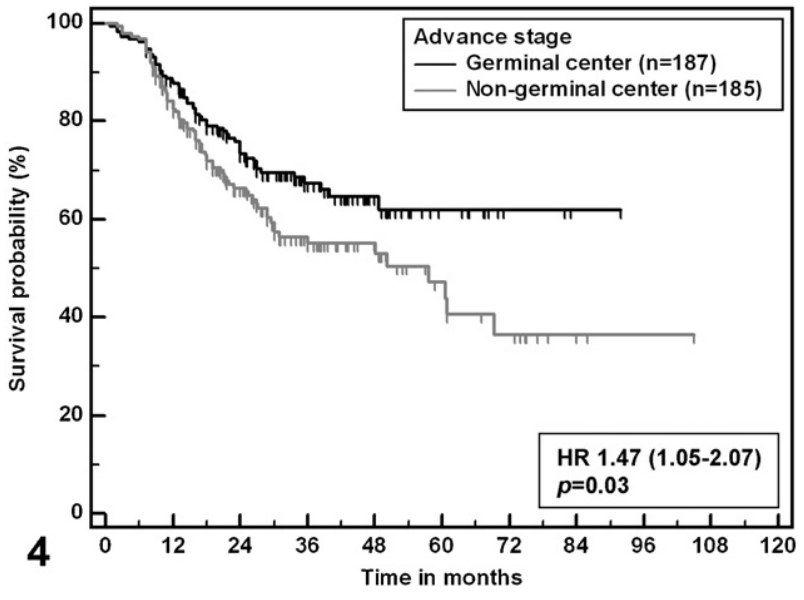

Fig. 4. Overall survival estimates in 372 patients with advanced stage diffuse large B-cell lymphoma treated with R-CHOP, according to the Hans algorithm.

[15]. One of the methods most commonly used is the Hans algorithm, which uses the expression of CD10, BCL6 and MUM1/IRF4 by the malignant DLBCL cells to classify patients as GC or non-GC [8]. However, the clinicopathological differences between GC and non-GC DLBCL patients as well as the prognostic and predictive value of the Hans algorithm have not been fully evaluated in the rituximab era.

Of most interest is that, according to the results of this study, there is a statistical association between GC profile and Asian ethnicity. Potential explanations for this finding include a true association, a systematic misclassification of GC or non-GC profile in Western or Asian patients, selection bias of Western or Asian patients or chance. Systematic misclassification and selection bias are unlikely, although not impossible, given that all the centers enrolled consecutive patients and followed the same criteria for classification of GC vs. non-GC profile. Two previous studies have investigated the racial differences between the incidence, presentation and outcome in patients with DLBCL $[16,17]$. However, none of these studies evaluated the IHC profile of these patients. The association between Asian race and GC DLBCL is a novel finding that should be further evaluated by molecular techniques.

Our study shows that the non-GC profile as defined by the Hans algorithm is associated with lower CR rates to R-CHOP in DLBCL patients. However, this potential predictive value does not translate into a prognostic one; although non-GC profile showed a statistical trend towards prognostic value for PFS and OS in patients with DLBCL treated with R-CHOP, it did not prevail in the multivariate analyses. The lack of prognostic value of the Hans algorithm is in concordance with other large studies in DLBCL patients [18], suggesting that more reliable tools need to be developed to sub-classify DLBCL patients. In fact, a recent study evaluated a series of algorithms, including Hans, and showed that a newer method, the Tally algorithm, was associated with a higher degree of concordance with genomic microarrays [19].

The predictive role of the Hans algorithm in the rituximab era has been previously evaluated in small studies with conflicting results $[11,20,21]$. A recent meta-analysis has shown a statistical trend towards an association between IHC profile and overall response rate [22]. However, a criticism is that this meta-analysis used study-level data and therefore could have been underpowered to identify smaller differences. Patient-level data, which were used in the present study, are intrinsically associated with less potential of bias introduction. Furthermore, although it is the authors claim, none of the studies included was a randomized controlled trial.

In the present study, we have attempted to critically evaluate the Hans algorithm in relationship to clinical and pathological characteristics as well as response to therapy and survival in newly diagnosed patients with DLBCL treated with R-CHOP. The large sample size of 712 patients in this study, the uniform therapeutic approach (all our patients received R-CHOP), and the multinational collaboration permitting the generalization of our findings, are all important strengths of our study. Although this is a retrospective study, which increases the risk of case selection bias, we have minimized it by contacting research groups that have evaluated consecutive patients. Furthermore, retrospective studies classically suffer from biases introduced by incomplete data. In this study, however, the clinicopathological data was complete in $99 \%$ of the cases included.

A weakness of this study is that there is a possibility our cases were misclassified, since there was not a centralized pathology review. However, our second look study evaluating $9 \%$ of our cases showed a strong inter-observer correlation on using the Hans algorithm $(r=0.85)$. The authors are aware of the reproducibility issues associated with IHC-based classification of DLBCL. In a recent study, the Lunenburg Lymphoma Biomarker Consortium evaluated 36 cases of DLBCL using an extensive battery of immunostains with the primary objective of evaluating inter-observer variation [23]. Such study, similar to ours, showed a high degree of reproducibility for CD10 but lower levels for BCL6 and MUM1/IRF4. It is important to notice that in our study there was a high variability among the reagents used to evaluate BCL6 expression among our institutions, which is likely the responsible of our lower rates of inter-observer correlation. Since there are data supporting prognostic value of BCL6 expression in DLBCL in the rituximab era [24], it is imperative to recognize that IHC-based risk-stratification of DLBCL, specifically with regard to BCL6 expression, should be evaluated in the context of studies with centralized pathology review. We believe the results of our study support the need of further evaluation and validation of markers for the IHC-based classification of DLBCL.

\section{Conclusions}

This study suggests that the Hans algorithm does not have prognostic value in DLBCL patients treated with R-CHOP; however, inter-observer reproducibility issues for IHC-based classification should be taken into account. An unexpected finding was that Asian populations might have a higher proportion of GC DLBCL. However, the latter should be confirmed by molecular analyses. Additional efforts should be directed at elucidating more reliable easy-to-use IHC-based algorithms to identify DLBCL subtypes in the rituximab era with future aims of not only risk-stratifying patients but also directing therapy to improve outcomes.

\section{Conflict of interest statement}

The authors have no conflict of interest to disclose.

\section{Acknowledgements}

Preliminary findings from this study were presented at Lymphoma \& Myeloma 2010: An International Congress on Hematological Malignancies in New York, New York, October 21-23, 2010, and the 52nd Annual Meeting of the American Society of Hematology in Orlando, Florida, December 4-7, 2010.

Contributors. Conception and design of the study: JJC; Acquisition, analysis and interpretation of data: JJC, BEB, MKS, II, SL, HN, RS, SU, JML, DOT, DS, JNB; Manuscript writing: JJC, JNB; Final approval of the manuscript: JJC, BEB, MKS, II, SL, HN, RS, SU, JML, DOT, DS, JNB. 


\section{References}

[1] Stein H, Warnke R, Chan W, et al. Diffuse large B-cell lymphoma, not otherwise specified. In: Swerdlow S, et al., editors. WHO classification of tumours of haematopoietic and lymphoid tissues. Lyon, France: IARC; 2008. p. 233-7.

[2] Feugier P, Van Hoof A, Sebban C, et al. Long-term results of the R-CHOP study in the treatment of elderly patients with diffuse large B-cell lymphoma: a study by the Groupe d'Etude des Lymphomes de l'Adulte. J Clin Oncol 2005;23:4117-26.

[3] Pfreundschuh M, Trumper L, Osterborg A, et al. CHOP-like chemotherapy plus rituximab versus $\mathrm{CHOP}$-like chemotherapy alone in young patients with goodprognosis diffuse large-B-cell lymphoma: a randomised controlled trial by the MabThera International Trial (MInT) Group. Lancet Oncol 2006;7:379-91.

[4] Lenz G, Wright G, Dave SS, et al. Stromal gene signatures in large-B-cell lymphomas. N Engl J Med 2008;359:2313-23.

[5] A predictive model for aggressive non-Hodgkin's lymphoma. The International Non-Hodgkin's Lymphoma Prognostic Factors Project. N Engl J Med 1993;329:987-94.

[6] Alizadeh AA, Eisen MB, Davis RE, et al. Distinct types of diffuse large Bcell lymphoma identified by gene expression profiling. Nature 2000;403: 503-11.

[7] Rosenwald A, Wright G, Chan WC, et al. The use of molecular profiling to predict survival after chemotherapy for diffuse large-B-cell lymphoma. N Engl J Med 2002;346:1937-47.

[8] Hans CP, Weisenburger DD, Greiner TC, et al. Confirmation of the molecular classification of diffuse large B-cell lymphoma by immunohistochemistry using a tissue microarray. Blood 2004;103:275-82.

[9] Song MK, Chung JS, Seol YM, et al. Influence of low absolute lymphocyte count of patients with nongerminal center type diffuse large B-cell lymphoma with R-CHOP therapy. Ann Oncol 2010;21:140-4.

[10] Seki R, Ohshima K, Fujisaki T, et al. Prognostic impact of immunohistochemical biomarkers in diffuse large B-cell lymphoma in the rituximab era. Cancer Sci 2009;100:1842-7.

[11] Ilic I, Mitrovic Z, Aurer I, et al. Lack of prognostic significance of the germinalcenter phenotype in diffuse large B-cell lymphoma patients treated with CHOPlike chemotherapy with and without rituximab. Int J Hematol 2009;90:74-80.

[12] Uccella S, Placidi C, Marchet S, et al. Bcl-6 protein expression, and not the germinal centre immunophenotype, predicts favourable prognosis in a series of primary nodal diffuse large B-cell lymphomas: a single centre experience. Leuk Lymphoma 2008;49:1321-8.
[13] Nyman H, Adde M, Karjalainen-Lindsberg ML, et al. Prognostic impact of immunohistochemically defined germinal center phenotype in diffuse large B-cell lymphoma patients treated with immunochemotherapy. Blood 2007;109:4930-5.

[14] Xia ZG, Xu ZZ, Zhao WL, et al. The prognostic value of immunohistochemical subtyping in Chinese patients with de novo diffuse large B-cell lymphoma undergoing CHOP or R-CHOP treatment. Ann Hematol 2010;89:171-7.

[15] Choi WW, Weisenburger DD, Greiner TC, et al. A new immunostain algorithm classifies diffuse large B-cell lymphoma into molecular subtypes with high accuracy. Clin Cancer Res 2009;15:5494-502.

[16] Shenoy PJ, Malik N, Nooka A, et al. Racial differences in the presentation and outcomes of diffuse large B-cell lymphoma in the United States. Cancer 2010 [Epub ahead of print].

[17] Griffiths R, Gleeson M, Knopf K, Danese M. Racial differences in treatment and survival in older patients with diffuse large B-cell lymphoma (DLBCL). BMC Cancer 2010;10:625.

[18] Ott G, Ziepert M, Klapper W, et al. Immunoblastic morphology but not the immunohistochemical GCB/nonGCB classifier predicts outcome in diffuse large B-cell lymphoma in the RICOVER-60 trial of the DSHNHL. Blood 2010;116:4916-25.

[19] Meyer PN, Fu K, Greiner TC, et al. Immunohistochemical methods for predicting cell of origin and survival in patients with diffuse large B-cell lymphoma treated with rituximab. J Clin Oncol 2011;29:200-7.

[20] Xia Y, Li ZM, Shi YX, et al. Short-term efficacy of rituximab-CHOP and CHOP regimens on two subtypes of diffuse large B-cell lymphoma. Ai Zheng 2009;28:146-9.

[21] Zinzani PL, Broccoli A, Stefoni V, et al. Immunophenotype and intermediatehigh international prognostic index score are prognostic factors for therapy in diffuse large B-cell lymphoma patients. Cancer 2010;116:5667-75.

[22] Fang C, Xu W, Li JY. A systematic review and meta-analysis of rituximabbased immunochemotherapy for subtypes of diffuse large B cell lymphoma. Ann Hematol 2010;89:1107-13.

[23] de Jong D, Xie W, Rosenwald A, et al. Immunohistochemical prognostic markers in diffuse large B-cell lymphoma: validation of tissue microarray as a prerequisite for broad clinical applications (a study from the Lunenburg Lymphoma Biomarker Consortium). J Clin Pathol 2009;62:128-38.

[24] Winter JN, Weller EA, Horning SJ, et al. Prognostic significance of Bcl-6 protein expression in DLBCL treated with CHOP or R-CHOP: a prospective correlative study. Blood 2006;107:4207-13. 